\title{
XXXV.
}

Aus dem Augustahospital in Breslau.

\section{Ueber eine Hernia traumatica sacralis nebst Bemerkungen zur Mastdarmplastik.}

\author{
Von \\ Privatdocent Dr. Alexander Tietze.
}

(Mit 2 Abbildungen.)

Unter dem Namen Hernia traumatica sacralis möchte ich im Folgenden eine Affection beschreiben, welche ich nach einer nicht völlig nach Wunsch gelungenen Mastdarmexstirpation mit temporärer Kreuzbeinresection beobachtet habe. Die Bezeichnung trifft jedenfalls das Wesen der Sache, wenn sie vielleicht anch durch eine geschicktere ersetzt werden kann.

Im Februar 1899 wurde von mir der damals 61 Jahre alte Herr L. aus Breslau im Augustahospital operirt. Es handelte sich um ein $4 \mathrm{~cm}$ uber dem Anus beginnendes Rectumcarcinom an der Vorderwand, etwa $3 / 4$ der Circumferenz einnehmend, von ca. $4 \mathrm{~cm}$ Längsausdehnung. Beschwerden hatten seit 3 Monaten bestanden. Der Tumor war verschieblich, Metastasen waren nicht nachzuweisen, die Resection des Mastdarms mit Erhaltung des Sphincter entschieden gut ansfuhrbar. Dieselbe wurde mit temporärer Kreuzbeinresection in der Weise vorgenommen, dass der Knochen mit einem V-förmigen Schnitt umgangen und das Kreuzbein in der Höhe zwischen 3. und 4. Kreuzbeinloch durchmeisselt wurde. Aufklappung des Hautknochenlappens, Resection des Darmes im Gesunden. Der heruntergezogene Darm wird zu etwa der reichlichen Hälfte seiner Circumferenz mit dem vorderen Theil des unteren stehen gebliebenen Restes vereinigt, der hintere Theil des Darmlumens bleibt offen. Peritoneum nicht geschlossen, sondern mit Jodoformgazebeuteln versehen, die aus der nur lose genähten Wunde herausgeleitet werden. Das offene Darmlumen mit lockerer Krüllgaze umgeben. Trotzdem Patient 8 Tage lang in passender Weise vorbereitet worden war, erfolgt am zweiten Tage Stuhl, welcher zu einer, glucklicher Weise in mässigen Grenzen sich haltenden Wundinfection Veranlassung giebt. Leider wird aber dadurch der knöcherne Antheil des bei der Operation gebildeten Lappens nekrotisch, 
Ueb. eine Ilernia traumatica sacralis nebst Bemerkungen z. Mastdarmplastik. 571

der Weichtheilrest rollt sich ein und schrumpft nicht unerheblich. Die Darmnaht luäit; soweit der Darm nicht genäht war, verwachsen die freien Ränder lippenförmig mit der Nachbarschaft. Nachdem innerhalb mehrerer Wochen die Heilung per granulationem erfolgt war, besteht ca. $3 \mathrm{~cm}$ uber dem Anus eine für 2 Finger bequem durchgängige Oeffnung, aus der sich aller Stuhl entleert und die Darmschleimhaut leicht prolabirt. Continenz besteht nicht. Der Patient verlangt dringend die Schliessung der Fistel, die im Juni desselben Jahres in folgender Weise vorgenommen wurde :

Der Darm wird hart am Rande der Schleimhaut umschnitten, die Darmwand von der Umgebung abgelöst und diese Ablösung an dem Kreuzbeinrande soweit durchgefuhrt, dass der obere Theil des Darmes ohne jegliche Spannung heruntergezogen und zur Vereinigung mit dem gegentherliegenden Darmende gebracht werden kann. Jetzt wird der Darm mit Lembert'schen Nähten in einer von links nach rechts verlaufenden geraden Linie geschlossen, die jetzt frei liegende Hinterwand, welche innerhalb eines dreieckigen Wundbezirkes liegt, mit zwei viereckigen, in ihrer Basis nahe dem Anus liegende Hautlappen aus der Nachbarschaft gedeckt. Die Entnahmestellen der Lappen werden, wie ublich, verkleinert, die Hautlappen an ihrer Berlhrungsstelle mit einigen Nähten aneinander befestigt, von den offen gelassenen Seitenschnitten wird je ein kleiner Streifen Jodoformgaze bis an die Nahtstelle locker unter die Hautlappen eingelegt.

Es wurde also mit anderen Worten die Fistel nach allgemeinen Regeln umschnitten, der obere Darmtheil stark mobilisirt, heruntergezogen, mit dem unteren Darmtheil quer vernäht und die Naht durch Hautlappen gedeckt. Heilung ohne $Z$ wischenfall. Die Fistel ist nun vollkommen geschlossen. $\mathrm{Pa}-$ tient hat ein vollkommen normal functionirendes Rectum, das nur an der Nahtstelle des Darmes leicht verengt ist. Dieser Zustand hält bis jetzt, also $31 / 4$ Jahr nach der Mastdarmresection, an, ein Recidiv ist nicht vorhanden, und Patient wilide absolut einem gesunden Menschen gleichen, wenn sich nicht nach und nach an der Operationsstelle eine eigenthumliche Hernie entwickelt hätte.

Schon wenige Wochen nach der Heilung der zweiten Operation begann die ganze bedeckte Partie sich hervorzuwölben, erst in geringem Umfange, bei Hustenstoss und Pressen nur wenig hervortretend, allmählich aber immer stärker und deutlicher. Eine bruchbandartige Bandage, welche dem Patienten verordnet wurde, war ihm zu lästig, und so entwickelte sich der Bruch unaufhaltsam bis zu seiner jetzigen Grösse.

Die Verhältnisse, die zur Zeit bestehen, sind folgende: Bei dem stehenden Patienten sieht man oberhalb des Afters eine calottenartige Geschwulst sich hervorwölben, deren Querdurchmesser $24: 28 \mathrm{~cm}$ und deren Höhe $6 \mathrm{~cm}$ beträgt. An der Hautbedeckung kann man noch gut als ein gleichseitig dreieckiges Feld den ursprïnglichen Hautlappen erkennen, der bestimmt gewesen war, mitsammt dem nekrotisch gewordenen Theil des Kreuzbeins die Beckenhöhle von hinten zu verschliessen, auch sind die Narben von der Plastik, im Ganzen in Form eines T, mit verlängerten seitlichen Schenkeln noch deutlich zu identificiren, doch erscheinen dieselben in allen Dimensionen enorm auseinander gezogen. Ibre Dichtigkeit hat dadurch, namentlich an den beiden seitlichen Schenkeln, so er- 
heblich gelitten, dass sie nur wie ein zarter Schleier die darunter liegende Intestina bedecken und ihre Contouren dentlich hervortreten lassen.

Beim Bücken und Pressen wölbt die Bruchgeschwulst sich viel deutlicher hervor: dasselbe geschieht explosionsartig bei einem Hustenstoss. In diesem Moment vergrössern sich mit einem Male sämmtliche Durchmesser um $2-3 \mathrm{~cm}$.

Der Percussionsschall war über der Geschwulst bei den letzten Untersuchungen (4 Stunden nach dem Mittagessen) absolut gedämpft. Die Bruchgeschwulst enthielt

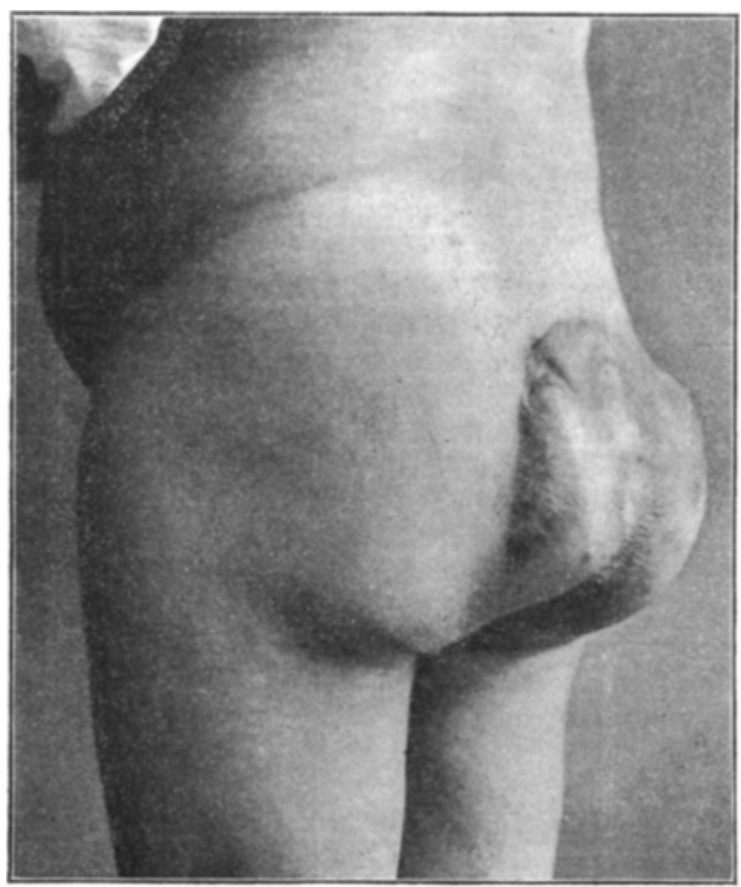

lïg. 1.

Patient ruhig stehend. vollkommen contrahirte Dünndarm. schlingen, welche man sehr deutlich im Bereich der seitlichen Narben in dem Moment erkennen konnte, wo der Patient Knieellenbogenlage einnahm. In diesem Augenblicke begann der ganze Inhalt der Bruchgeschwulst in das Innere des $\mathbf{A b}$ domens hineinzutreten: schnell sah man unter der dunnen Narbe die eng contrahirten Därme uber den Rand der Bruchöffinung zuritckgleiten, und ehe man sich dessen versah, blieb an Stelle der früheren Erhabenheit eine tiefe Einsenkung zurtick, die bequem eine

kräftigeMännerfaust aufnehmen konnte. Man fühlte jetzt die Bruchpforte in Gestalt eines liegenden Rechteckes vom Durchmesser $4: 9 \mathrm{~cm}$, dessen oberer Rand vom Kreuzbein, dessen übrige Seiten von derben Muskelund Narbenrändern gebildet wurden.

$\mathrm{Zu}$ diesem Krankbeitsbericht ist Folgendes zu bemerken:

Zweifellos ist die Entstehung dieser sacralen Hernie auf das Missgeschick zurückzuführen, dass das „temporär" resecierte Kreuzbeinende nekrotisch geworden war. Glücklicher Weise scheint dieses Ereigniss recht selten $\mathrm{zu}$ sein, wenigstens vermisst man derartige Notizen in der Literatur. $O b$ die von mir gewählte Methode der Aufklappung, deren 
sich auch He gar für seine Zwecke in vielen Fällen bedient hat, unsicherer sei, als die anderen Methoden temporärer Kreuzbeinresection, kann ich nach meinem Material nicht entscheiden. Wenn es zu Infection kommt, dann ist das Schicksal des Knochens selbstverständlich gefährdet, mag man operiren, nach welcher Methode man wolle. Thatsächlich habe ich unter 6 Fällen gleicher Art die Nekrose des Knochens nur eben dies eine Mal erlebt. Dementsprechend ist es auch niemals wieder zur Bildung der eigenthümlichen hernienartigen Hervorwölbung gekommen. Einer dieser Fälle war dagegen insofern dem oben beschriebenen ganz gleich, als sich gleichfalls am Ende des replantirten Lappens eine weite Darmfistel etablirt hatte, die nach einigen Monaten plastisch geschlossen wurde, ganz wie in dem Falle L. Somit trägt für diese Ausführung der Mastdarmresection sowohl die Stelle der Etablirung der Fistel (an der Spitze des Hautkreuzbeindreiecks), als auch der spätere plastische Verschluss derselben einen gewissen typischen Charakter. Die zuletzt erwähnte Operation betraf diesmal eine Frau: ich habe sie im Jahre 1896 operirt und noch in diesen Tagen von dem Hausarzt erfahren, dass sie völlig gesund ist und dass ihr Darm functionirt, wie bei einer ganz normalen Person. Ich selbst habe sie vor 2 Jahren das letzte Mal untersucht und an der Stelle der Darmvereinigung eine ganz leichte Stenose gefunden, sonst aber alles in Ordnung.

Auch sonst war ich noch mehrmals in der Lage, plastische Operationen am Rectum auszuführen, bei denen es sich meist darum handelte, Fisteln zu schliessen, die nach Mastdarmexstirpationen von mir oder andern übrig geblieben war. Hier musste man sich nach verschiedenen Regeln der Plastik helfen: einen gewissen gemeinsamen Typus repräsentirten nur die Fälle, die man nicht selten nach der ursprünglichen $\mathrm{Kr}$ aske'schen Methode sah, wenigstens wie wir sie an der Klinik meines hochverehrten Lehrers v. Mikulicz früher ausführten. Indem wir damals den hinteren Rand des Darmes nicht vernähten, vielmehr den unteren Theil noch in der Mittellinie spalteten, erlebten wir nicht selten, dass das obere Darmende sich umkrempelte, in die Höhe schlug und mit dem wunden Kreuzbeinrande verwuchs. Wahrscheinlich haben andere Operateure dasselbe gesehen. Mit Recht wurden diese Fälle von uns und den behandelnden Collegen als ganz besonders unangenehm empfunden. Man batte eine lange, leicht verletzliche, nach hinten offene Schleimhautrinne vor sich; von einer Continenz, ja auch von dem Anbringen eines einigermaassen sicheren Verschlussapparates war keine Rede; die Patienten waren über ihren Zustand meist höchst deprimirt; und meldete sich dann in einiger 
Zeit das Redidiv, so begriff man die Abneigung des betreffenden Hausarztes, in einem zweiten Falle überhaupt operiren zu lassen. Thatsächlich hält ja auch jetzt noch die Furcht vor der schlechten Function nach der Heilung sehr viele Collegen davon ab, ihre Patienten sobald als möglich zum Chirurgen zu bringen, und wenn wir auch diesen Standpunkt nicht billigen können, einfach deswegen nicht, weil sich bei der Mastdarmexstirpation auch unsere functionellen Resultate ganz erheblich gebessert haben, so ist es doch ganz richtig, dass die Rücksicht auf die spätere Function ganz wesentlich die Frage beeinflusst, wie weit wir die Grenzen eines operativen Eingreifens ziehen dürfen. Selbst die Gefahr eines Recidivs verliert

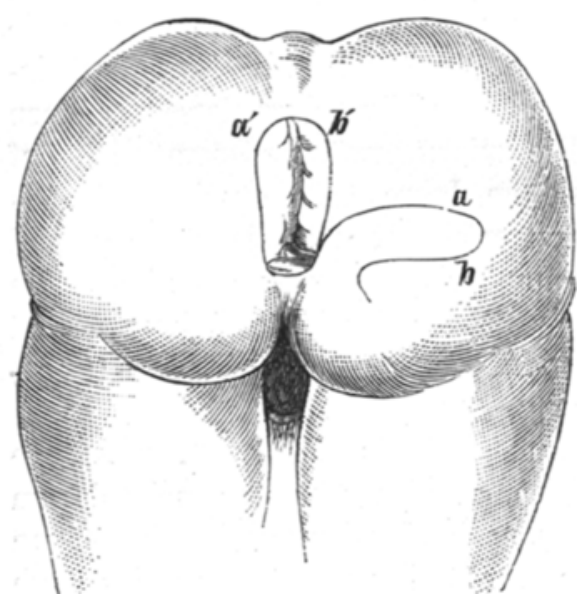

Fig. 2. etwas von ihrer traurigen Bedeutung, wenn wir wenigstens in der Zwischenzeit unseren Patienten ein beschwerdefreies oder doch erträgliches Dasein geschaffen hatten.

So war es nun freilich bei den oben skizzirten Verhältnissen nicht. In einem dieser von mir operirten Fälle habe ich ein leidliches Resultat in folgender Weise erzielt:

Es handelte sich um einen Patienten, bei dem der untere Theil des Kreuzbeins und das Steissbein von einem Längsschnitt aus entfernt war. Ebenso war der Sphincter entfernt worden. Der vordere Rand des heruntergezogenen Darmes war in der Höhe des Analringes angenäht worden und dort festgewachsen, der hintere Kand hatte sich in die Höhe geschlagen und sass am Kreuzbein fest. Fs bestand also jene oben geschilderte, nach hinten offene Darmrinne. Dieselbe wurde in ihrer oberen Hälfte umschnitten, der Darm so weit abgelöst, dass wieder dieser Theil heruntergeklappt werden konnte, und so ein Darmrohr entstand, das freilich an seiner Hinterwand unbedeckt war. Da dasselbe sich zu weit erwies, so wurde es durch eine Reihe in der Mittellinie angelegter Catgutnähte längsgefaltet und verengert, dann durch einen Lappen aus der Nachbarschaft in der Art beistehender Figur bedeckt. - Mit dem Peritoneum kam ich hier ebensowenig wie in einem anderen Falle von Mastdarmplastik in Conflict.

Das Resultat bestand darin, dass der Patient einen allerdings sphincterlosen Anus an normaler Stelle erhielt. Auch er war nach $31 / 2$ Jahren ich habe ihn seitdem aus dem Gesicht verloren - noch recidivfrei und Latte fllr derben Stuhl eine ganz befriedigende Continenz. 
Ueb. eine Hernia traumatica sacralis nebst Bemerkungen z. Mastdarmplastik. $\mathbf{6 7 5}$

Die bei den bisher erwähnten Patienten erzielten Dauerheilungen entsprechen nun leider nicht der allgemeinen Statistik meiner Mastdarmexstirpationen: sie sind vielmehr unter einem allerdings nur kleinen Materiale - dreissig - die einzigen so glücklich verlaufenen. Dass ich sie hier anführte, lag daran, dass natürlich für nachträgliche Plastiken im Wesentlichen die günstigen Fälle das Material liefern werden.

Uebrigens ist ja durch manche Verbesserungen der Technik, vor allen Dingen das Nikoladoni'sche Verfabren, neuerdings das Gebiet der plastischen Bestrebungen glücklicher Weise erheblich eingeschränkt worden, und gerade aus der v. Mikulicz'schen Klinik ${ }^{1)}$ konnte ja über die durch jene Methode erreichten Fortschritte berichtet werden. Ein weiterer Fortschritt liegt nach meiner Ansicht darin, dass man in allen Fällen, wo der Sphincter nicht erhalten werden konnte, auf alle anderen Versuche verzichtet und principiell den sacralen After anlegt, natürlich nach dauernder Kreuzbeinresection im Sinne der ursprünglichen Kraske'schen Methode. Auf die Vorzüge des Verfahrens hat bereits $\mathrm{H}$ ochenegg hingewiesen. Ich habe jetzt gleichzeitig 3 Patienten dieser Art in Beobachtung, die von mir vor $5 / 4$ Jahren, $3 / 4$ Jahren und 3 Monaten operirt worden sind (bisher recidivfrei). Mit einer einfachen Bandage, wie sie auch Hochenegg angegeben hat, fühlen sich diese Patienten sehr wohl und gehen ihren Geschäften nach, so dass sie durchaus mit ihrem Zustand zufrieden sind. Bei allen dreien haben sich die Verhältnisse so geregelt, dass täglich einmal Stuhl erfolgt. Nur bei einem der Patienten ist ein mässiger Darmprolaps vorhanden. Man hätte eine Entstehung desselben vielleicht durch eine Gersuny'sche Drehung oder durch eine anderweitige Verengerung der Ausgangsöffnung vorbeugen können. Rechnet man hierzu die Vereinfachung der Wundverhältnisse bei dieser Methode, so kann man mit derselben sehr zufrieden sein.

In die Verlegenheit, Mastdarmplastiken ausführen zu müssen, ist gewiss jeder Chirurg vielfach gelangt. ${ }^{2}$ ) Da aber von dieser Angelegenheit in der Literatur, wie bereits bemerkt, sehr wenig die Rede ist, so mochte die kurze Mittheilung obiger Fälle, gerade weil sie ein im gewissen Sinne typisches Operationsverfahren illustriren, gestattet erscheinen.

1) Sieber, Leber die Befestigung des Rectums nach $\mathrm{Nik}$ ola d o ni. In.-Diss. Breslau 1900.

2) Vergleiche: Stiassny, Ueber plastische Nachoperationen nach Resectio recti. Brun's Beiträge. Bd. 29. S. 491. 